\title{
A Survey of Robotic Hand- Arm Systems
}

\author{
C.V. Vishal Ramaswamy \\ B.E third year, Department of Computer Science \\ and Engineering, \\ SSN College of Engineering, \\ Chennai
}

\begin{abstract}
From the last three decades creating human robotic hand replica with enhanced capabilities is of concern and lot of efforts have been put into it. This paper focuses on understanding the different techniques that are used for human robot interaction in robotic hand arm systems. Diversification is stated in areas of human and robotic hand interaction, the degrees of freedom, the grasping ability, number of fingers and materials used for the hand. The flexibility of grasp is compared in terms of Degrees Of Freedom (DOF) and the number of finger end effectors. The controlling method is either through sensor based or gesture controlled or simulation based or pre-defined positions.
\end{abstract}

\section{Keywords}

Robotic hand, Robotic arm, Human hand replica, Human robot interaction.

\section{INTRODUCTION}

Since the inception of robots, (the name came from the word rabu meaning slave) humans have been trying to transfer human capabilities to robots for easier and enhanced work to be conducted. In this regard, one of the most important parts of our human body, our hands are replicated as robotic hands. The hand is one of the most complex and load bearing part of our human body which acts as an input(tactile sensing) as well as output(physical work) device to humans. Lot of research has and is being done in medical field to develop a prosthetic hand with enhanced capabilities. In this regard the field of robotics has also explored the depths in creating a robotic hand that mimics human or in even better ways.

Tele operated robotic hands that mimic human hand are very much useful in areas of research, defence, hazardous chemical industries, factories, etc. where humans have great threat and thus is the growing field of research in this era.

The more, the flexibility and dynamic control of robotic hands, the more is the complexity of design. In the following section we will discuss the various robotic hand that have stormed the robotic field and state a clear distinction and comparison of these their pros and cons.

\section{REVIEW OF ROBOTIC HANDS}

\section{1 iHY Robot Hand}

The iHY robot hand (see Figure 1) designed by iRobot, Harvard and Yale students is a design which had two fingers and an opposable thumb which were driven by five motors. Each finger contained proximal and distal links which connected finger to the base till the fingertip [1].To induce flexibility and durability, heavy duty elastic joints were used which provided added improved grasp around the objects. Objects ranging from small ball bearings, golf balls up to heavy drilling machines can be held by these iHY hands. The grip power is provided using cable tendons that wrap the object clutching it firmly.

\author{
S. Angel Deborah \\ Assistant Professor, Department of Computer \\ Science and Engineering, \\ SSN College of Engineering, \\ Chennai
}

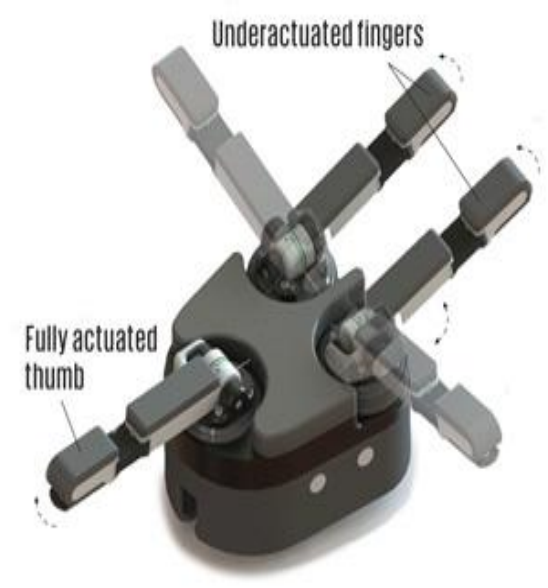

Figure 1 iHY Hand

Out of the five motors that control the three fingers, four are used to drive the fingers while the fifth motor allows fingers to move quickly from one orientation to another. An added part called the finger nail enables the pinch grasp feature for picking cards; tiny balls etc. (see Figure 2).

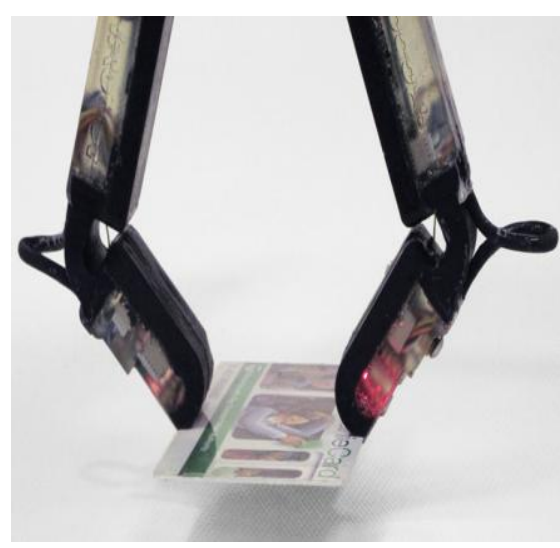

Figure 2 Pinch grasp

Fibre optic sensors were used to track the motion of the object that is held in the hand. The fibre optic cables emitted light depending on the variation in the angle of the joint .Thus the receptors of the light identify the amount of bending that the fingers have undertaken. The pressure sensors are utilised to indicate how strong and powerful the grasp should be. Each finger has 22 and the palm has 48 pressure sensors which are connected to PCB board. Each finger has a separate microcontroller embedded in it which collects data from joint and touch sensors, send it to the computer through the Ethernet for manipulation and actuates the finger to perform accurate grasp. 


\subsection{I-Limb Ultra}

The i-limb ultra (see Figure 3) is a prosthetic hand that is designed for people who need more prosthesis. With i-limb ultra the prosthesis moves and looks like a natural hand [2].

The i-limb ultra is controlled by software called biosm that excels in providing customizable hand postures that are needed for day to day lives. 14 pre defined automated grips are available for choice to complete daily chores like the index finger mode, pinching mode etc.

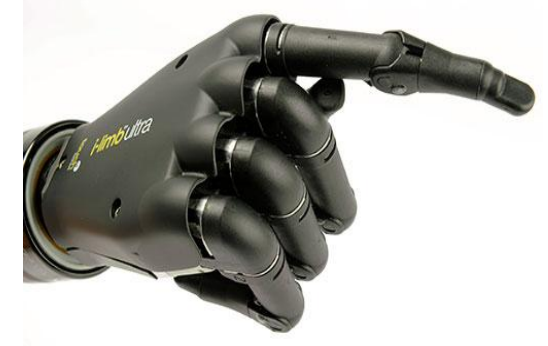

Figure 3 I-Limb Ultra

It also has the vari-grip feature, that allows user to have an extra strong grip like when tying shoelaces or opening a bottle cap etc. [2]. The robotic hand has five fingers with the thumb which can be manually rotated to adjust the grasping position. Mobile app can be used to switch between the 14 configurations that are pre defined. Another feature is the auto-grasp that prevents the objects from slipping and the hand automatically moves to the natural position after period of inactivity.

\subsection{Hand Gestures Controlled Robotic Arm}

This model deals with designing a robotic hand that mimics human hand. It is composed of sensors and actuators which are connected by XBEE module through arduino uno board.

The Arduino IDE is used for manipulation operations for analysing the sensor inputs and producing the motor driving outputs. The programs are coded in $\mathrm{C} / \mathrm{C}++$ language. [3] The robotic glove controller is the sensory controller which houses the sensors that track the movements of human hand. Five flexion sensors are attached to the glove, one for each finger. The resistance changes according to the variation in bending, and the resistance change is read as analog input by the Arduino Uno. The resistance values are tabulated and corresponding manipulations are done. The robotic arm possesses five servo motors for controlling the finger movement and three servos for pan and tilt motion. These servo motors receive the driving angle from the Arduino uno connected to it.

The main modification in this method is the use of XBEE modules for communicating between the two Arduino uno boards .The XBEE modules, the transmitter and receiver are attached to the glove and the robotic hand respectively and integrate by radio frequency (RF) communication

\subsection{Shadow EDC Hand}

The Shadow EDC ( Ether CAT Dual Can) hand (see Figure 4) developed by Shadow Robot Company is the most advanced Dextrous hand which can be used as a tele-operation tool or mounted on a range of robot arms as a part of robot system[4]. The essential features include the Human Kinetics, High Bandwidth sensing, BioTac Tactile sensors, etc.

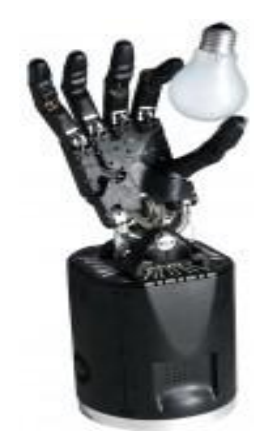

Figure 4 Shadow Hand

The Shadow hand has 20 actuated degrees of freedom and 4 under actuated movements for 24 joints. These joints are actuated to provide the exact human motion including the flexion motion of the palm and the thumb. This model has a total of 129 sensors which enable to manipulate the control system and understand the environment. The position and force sensing are also done which along with the tactile sensing data are sent using high bandwidth $100 \mathrm{~Hz}$ to $1 \mathrm{KHz}$ through Ether CAT interface. For providing high sensing abilities like force, micro vibration and temperature gradient, sensing was implemented using tactile sensors and transferred using the Ether CAT.

The most important advancement was the algorithm devised for grasp stabilisation and control (GSC) [4]. Based on the point cloud 3D value, segmentation algorithm was applied, which searches for a pre computed grasp and it is implemented. If the object to be grasped is not known and its grasp is not yet computed then a set of possible grasps are collected and the mesh is reconstructed over it.

\subsection{Analysis of Hand Arm System using Electro Goniometer}

In this model the artificial hand is actuated by command control equipment which uses an electro goniometer to track the hand movements. The electro goniometer is made of one potentiometer and two rods; one of them is fixed on the longitudinal axis of ulna bone, while the other axis is mobile, executing the flexion-extension movement in the same time with the hand. The rods are fixed on the forearm and hand with elastic straps, type Velcro [5]. The values recorded from the potentiometer are transferred to the PC for computation using the Arduino Mega2560 and processed in $\mathrm{C} / \mathrm{C}++$. The manipulated values are transferred to the artificial hand using Bluetooth module. The artificial hand had flexion extension actuators for the fingers and the thumb rather than motors to avoid skeletal disorders (see Figure 5).

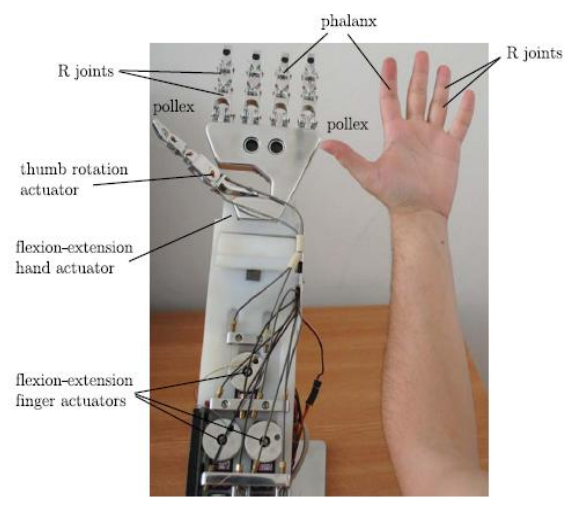

Figure 5 Artificial hand 
The main study was related to wrist joint movement and its normal and abnormality from a series of test results, then the Largest Layout Exponent (LLE) was calculated .It was found that the mean LLE of the wrist movement was approximately from 0.020 to 0.032 . Based on these results the exact movement of human wrist was replicated to the artificial robotic hands.

\subsection{The Handroid}

The Handroid prototype (see Figure 6) was developed by ITK Japan which is a remotely operated five finger movable robot hand. It can be used in engineering environments which are inaccessible and dangerous to human hands [6].

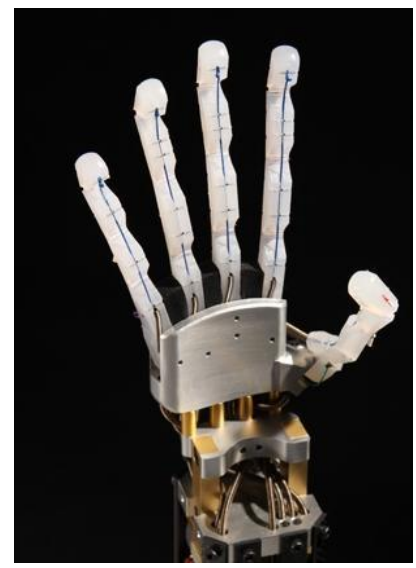

Figure 6 The Handroid

This model has four fingers and a thumb with 5 Degrees Of Freedom in each finger, which adds up to 15 DOFs on the whole and thus, can replicate the exact movements of human hand. [6]. These movements are produced using 6 motors connected to the wrist, out of which 4 are used to control the fingers and 2 for the thumb. Important advancement in this model is the inclusion of fluid motion to provide better control. The robot hand is made with lightweight aircraft parts and includes the advance metalworking techniques [6]. The robot hand model developed by ITK is controlled by a master slave correspondence between human hand and robotic hand.

The sensory gloves worn on the human hand acts as the master which remotely controls the robotic hand. When the user performs any movements, the sensors in the gloves calculates the changes in motion and sends the control signals to the controller connected to the slave.

\subsection{Real Time Robotic Hand Control Using Hand Gestures}

This paper proposes a robotic hand model that is controlled in real time by image capturing and processing of the actual hand movement. Image processing tools like MATLAB are used for capturing the image, normalising, and for noise reduction. A systematic approach is followed which includes image acquisition, hand gesture extraction, determining hand pattern using Principal Component Analysis(PCA) algorithm and converting them into instructions to control the robotic hand.

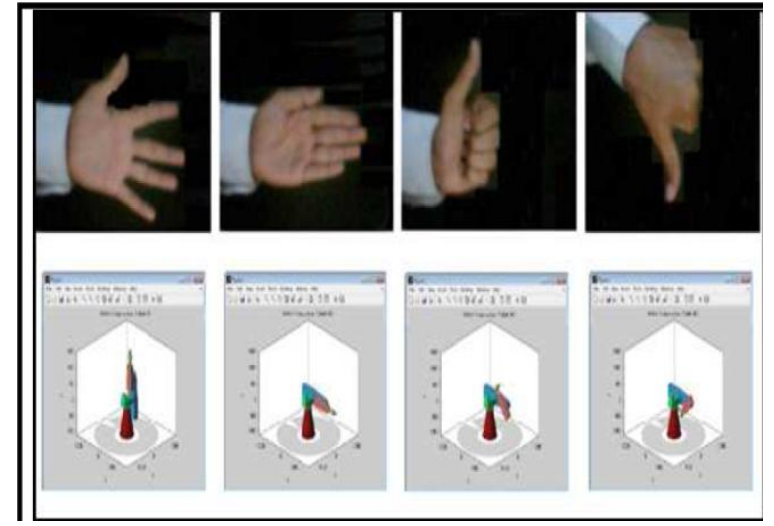

Figure 7 Gesture database

The PUMA robotic model was chosen to implement the motion traced by the human hand. A gesture database consisting of binary images of size $60 \times 80$ pixels is pre stored, so it takes less time and memory space during pattern recognition.[8] Whenever a gesture is matched with a meaningful gesture from the database,(see Figure 7) the instruction set corresponding to that gesture is identified and passed to robot for execution. Movement commands are written as a function in robot specific language. In this way the robotic system can be controlled by hand gesture using live camera [8]

\subsection{Multi Fingered Robotic Hand Controlled using Bluetooth Wireless Module}

This model is based on master slave control concept where the sensorized glove acts as master and the multi fingered hand acts as slave. The master controls the slave using KC-21 Bluetooth wireless module. The slave has 5 fingers with a total of 15 DOFs by $3 \mathrm{DOF}$ for each finger. The multi fingered hand was developed using 5 volt dc motors which are controlled by L293D motor drivers. The end effectors, the robotic hand also has flex sensors to act as a feedback mechanism for tracking the movement of the dextrous hand. To control the robotic hand PIC18F4520 18's family mid range microcontroller is used as controller.[9] The master (sensorized glove) has flex sensors, the bend sensing resistance which is worn on the human hand and senses the actual position and tracks the movement of fingers.

\subsection{Industrial ROBOT STAUBLI TX 90, the Mechanical Hand SAH with Collision Detection}

The Schunk Anthropomorphic Hand (SAH) used with the Industrial Robot Staubli TX 90developed by Jan Rosell Raul Suarez Carlos Rosales and Alexander Perez [10] was controlled by hand and robot simulator which captured the hand positions and movements with sensorized gloves. The SAH which is based on the DLR hand has three fingers with four joints and the thumb with five joints, thus there are a total of 17 joints with 13 independent DOF (see Figure 8) .The industrial robots Staubli TX 90 has six DOF. 


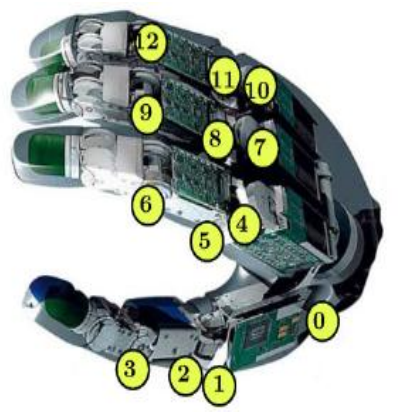

\section{Figure 8 Anthromorphic SAH Hand}

Cyber Glove (commercial sensorized glove) was used, which provides 22 joint angle measurements. Three flexion sensors are used per finger, four abduction sensors between fingers, a palm-arc sensor and two sensors to measure flexion and abduction of wrist. The simulator tracks the position and movements of both the sensory glove and the robotic hand.

The main feature of this setup is that it includes collision detection. The algorithm used for collision detection is a probabilistic roadmap planner that samples the different configurations and connects them together to find out the collision and thus dimensionally reduce the hand search space results in less computational times. The dimension of the hand search space is increased in iteration whenever there is no collision path for a particular configuration. [10] Thus if the solution cannot be found in the available sample space, the volume is increased incrementally to connect the initial and final configuration. It keeps track of the initial and final configurations of the components to find the initial and final distances from the centre of the hyper cube which defines the region of arm configurations. As the proposed algorithm had no critical parameters to be tuned, this model was efficient in developing a motion planner for hand arm robotic systems that could identify likelihood of human postures and obtain a grasp or pre-grasp configuration.

\subsection{A Five Fingered Robotic Hand Prototype by using Twist Drive}

This robotic hand has five fingers coupled with proximal interphalangeal joint (PIP) and the Dextral Interphalangeal Joint (DIP) (see Figure 9). The coupling is used to reduce the number of actuators while keeping the motion similar to that of human intact. The coupling ratio varies between the fingers and individuals, but it is measured in the range of 1.5 to 2 [14], [15].

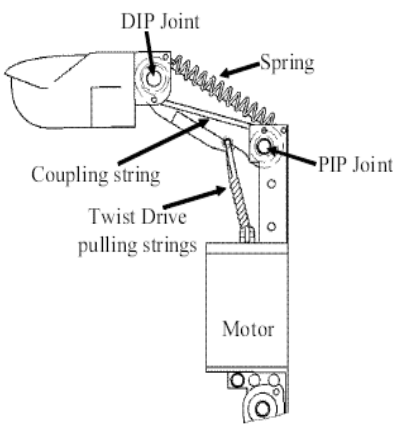

Figure 9 PIP and DIP coupled joint design

The number of degrees of freedom is limited to 18 of which 14 are independent, while the rest are coupled with DIP and
PIP joints. The hand has 15 small size brushless DC Servo motors and 15 channels servo controller drive electronics. The main advantage of design is total weight of the hand is 800 grams which is very less. Each finger is controlled by a separate microcontroller. Every controller board is interfaced with the Personal Computer using RS232 serial interface. The calculations are done by the PC and the electrical controlling of the fingers is done by the microcontrollers.

This system was mainly proposed to overcome the fact that the robotic hands are precise but stiff, accurate but expensive, durable but dangerous [11]. The main objective was to design zero impedance joints using twist drive. The drive mechanism was used to implement this, which overcame the existing drawbacks of artificial muscles like producing low force, slow response, etc.

\subsubsection{Twist Drive mechanism}

The Twist drive mechanism uses two strings that twists on each other to produce a pulling force but does not push. The joint can be set into Free State by just unwinding the twist.

The rotating movement of the shaft produces a lateral movement or pull to the driven object or when a torque $\mathrm{T}$ is generated on motor's shaft, a pulling force $\mathrm{F}$ is produced on the driven object (see Figure 10).

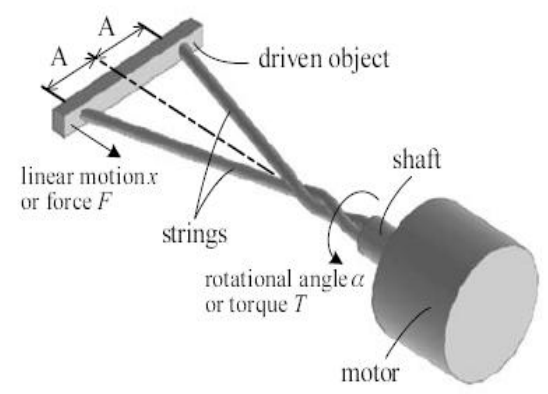

Figure 10 Twist Drive Principle of operation

\subsection{The Kinetic Humanoid Hand (KH)}

The Kinetic Humanoid type $S$ for sign language is a novel robot hand PC based master slave system which demonstrates the effectiveness in grasping and manipulating objects [12]. The $\mathrm{KH}$ robotic hand is a five fingered hand with 20 joints and 15 DOFs and uses DC motors and Servo motors to control the movement of fingers. The data glove (Cyber glove) is used for measuring the joint angle of the finger. A 3$\mathrm{D}$ position measuring device is used for measurement of hand position of user. An orientation tracking system is used to track the operator's hand posture. The important added feature in this model is the reduction of backslash and the introduction of tactile sensors.

\subsubsection{Reduction of backslash}

Each finger in a hand has two joints and the main objective of this model is to reduce the backslash produced by one joint on another. The lower the backslash the higher the friction of gears transmission [12]. To reduce backslash the torques at the joints are calculated, corresponding sine waves are generated. Then the root mean square values are computed to reduce the friction between the gears.

\subsubsection{Tactile sensors}

895 tactile sensors are distributed over the fingers, palm and the thumb to acquire the tactile information accurately. These 
sensors detect the contact positions and forces that are mounted on the robotic hand surface. The proportionality between the pressure and electrical resistance is the underlying concept of these tactile sensors. The tactile capability is used as a mechanism for manipulating the objects. The feedback is provided to the human hand (see Figure 11), using the feedback gloves which have servo motors that transmit torque to the fingertips.

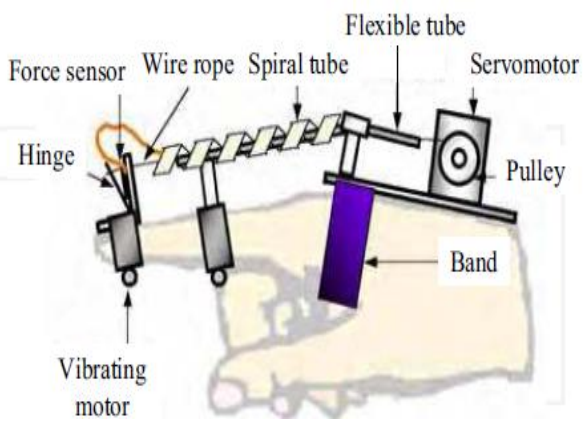

Figure 11 Force feedback mechanism

\section{CONCLUSION}

Through the review of the above papers, industrial models and books on robotic hands and their control, we can say that the field of robotics especially in mimicking human motions is growing and expanding rapidly. The various artificial models discussed above show differences in the number of fingers, the number of degrees of freedom, the methodology of control, the interface and ways of tracking hand movement. On an overall basis, majority of the models have a sensory glove, Cyber glove or force feedback glove that are worn on human hands to monitor and record the human hand movements. The robotic end effectors are generally five fingered and each model has some extra added feature like pressure sensing, tactile sensing, twist drive feature etc. Mostly the arduino IDE is used for processing the sensor information into the control signals for actuators, while the communication is established either through Ethernet, Bluetooth or RF communication. The models largely used the sensory gloves for control while other methods like using electro goniometer, hand gesture recognition using image processing was also used. In spite of all the advancement and expertise in this area, still the problem of efficiency, size, weight and speed of interaction between master and slave need to be improved, to be able to suit for real time dynamically changing environment. The future scope pertains to applying slight modifications to overcome these problems. By using micro servos, modelling using 3D printing, gesture control using image processing and long distance communication using high speed internet protocol, a better model can be developed which minimises or removes the before mentioned drawbacks. With the field of networking ever developing, the current internet protocol IPV4 in combination with IPV6 protocol can be used for long distance tale-operation.[7] The reflexivity and fast control of robotic hands with human hands can be done with high precision using advanced tools for image processing like MatLab, OpenCV etc., to capture and track human hand movements. With the use of micro servos, the required torque can be achieved with minimum weight and size, to make the robotic hand lighter and more like real hand. 3D printing and modelling provides the flexibility, strength and real hand like look. These robotic hands can be used in prosthesis as prosthetic hands, attached over the handicapped hand and by analysing the nerve pulses from the cut arm; the artificial hand can be controlled to function as the human hand which is the scope of future.

\section{REFERENCES}

[1] Inexpensive, Durable Plastic Hands Let Robots Get a Grip By Robert Howe, Aaron Dollar \& Mark Claffee Posted $21 \quad$ Nov 2014 http://spectrum.ieee.org/robotics/humanoids/inexpensivedurable-plastic-hands-let-robots-get-a-

grip/?utm_source=techalert\&utm_medium=email\&utm_ campaign $=120414$

[2] i-Limb ultra 2014 Touch Bionics Inc. and Touch Bionics Limited http://www.touchbionics.com/products/activeprostheses/i-limb-ultra

[3] Hand Gestures Controlled Robotic Arm Aditya Purkayastha, Akhil Devi Prasad, Arunav Bora, Akshaykumar Gupta, Pankaj Singh Journal of International Academic Research For MultidisciplinaryImpact Factor 1.393, ISSN: 2320-5083, Volume 2, Issue 4, May 2014

[4] Shadow Robot Company: http://www.shadowrobot.com/products/dexterous-hand/ 2014 Shadow Robot Company

[5] Analysis of a hand arm system Daniela Tarnita, Dan B. Marghitu Robotics and Computer-Integrated Manufacturing 29 (2013) 493-501

[6] ITK, Multi-fingered Robot Hands Handroid from:http://www.itkpro.com/en/pro/kindengisyu.htmITK.Japan "Handroid" Hyper Japan 2012

[7] Review of Control Mechanism of Multi-fingered Robotic Arm and Proposal of New Design Anand Kumar Kirori Prof. RajeshwarLalDuaIOSR Journal of Engineering May. 2012, Vol. 2(5) pp: 1251-1254

[8] Real-Time Robotic Hand Control Using Hand Gestures JagdishLalRaheja, RadheyShyam,G.Arun Rajsekhar and P. Bhanu Prasad Robotic Systems - Applications, Control and Programming, Published online 03, February, 2012 Published in print edition February, 2012 http://cdn.intechopen.com/pdfs-wm/27419.pdf

[9] Multifingered robot hand robot operates using teleoperation, MohdKhairulAkhwan Bin Ahmad Faculty of Electrical and Electronic Engineering Universiti Tun Hussein Onn Malaysia, JULY 2011

[10] Autonomous motion planning of a hand-arm robotic system basedon captured human-like hand postures Jan Rosell, Raul Suarez, Carlos Rosales, Alexander Perez, Autonomous Robots (2011), Vol. 31, No. 1.pp 87-102 https://www.ioc.upc.edu/personal/jan.rosell/publications/ papers/autonomous-motion-planning-of-a-hand-armrobotic-system-based-on-captured-human-like-handpostures

[11] A Five Fingered Robotic Hand Prototype by using Twist Drive, Ivan Godler, Takashi Sonoda, ISR / ROBOTIK 2010

[12] A Novel Anthropomorphic Robot Hand and its Master Slave System Tetsuya Mouri and Haruhisa Kawasaki Source: Humanoid Robots: Human-like Machines, Book 
edited by: Matthias Hackel ISBN 978-3-902613-07-3, pp. 642, Itech, Vienna, Austria, June 2007

[13] Robotic Hands: Design Review and Proposal of New Design Process Jimmy W. Soto Martell and GiuseppinaGin International Journal of Mechanical, Aerospace, Industrial and Mechatronics Engineering Vol: 1 No: 2, 2007
[14] M. Nakamura, C. Miyawaki, N. Matsushita, R. Yagi,Y. Hanada: Analysis of Voluntary Finger Movements During Hand Task by a Motion Analyzer, J. Electromyography and Kinesiology, Vol. 8, No. 5, 1998 ,pp. $295-303$

[15] Quantitative Analysis of the Linkage Between the Interphalangeal Joints of the Index Finger P. Hahn, H. Krimmer, A. Hradetzky, U. Lanz, Vol. 27, No. 4, 1994, pp. $479-491$ 